\title{
Cooling of the heating device in conditions of time-varying ambient temperature, heat transfer coefficient and radiation temperature of the fencing
}

\author{
A.G. Gulkanov, K.A. Modestov, R.G.Akhverdashvili \\ National Research Moscow State University of Civil Engineering, 26, YaroslavskoyeShosse, Moscow, 129337, \\ Russia
}

\begin{abstract}
Nowadays, there is no general method that takes into account the heat storage capacity of heating devices for calculating the cooling of buildings in the event of an emergency shutdown of heat supply. This work is devoted to finding the temperature at any time and the amount of heat released with time-variable parameters of the air temperature surrounding the heating device, the temperature of the heating device, the heat transfer coefficient and the temperature of the internal surfaces of fences.
\end{abstract}

Key words: athematical model, heating device cooling, emergency heat supply.

\section{INTRODUCTION}

In the modern analytical theory of heat stability, many scientific papers have been published, in which the problems of cooling (heating) objects with different properties, geometric shape and other parameters have been considered [8-13, 16-18]. In this paper, we consider the problem of cooling the heating device, which is of an applied nature. At the moment, there is no method for calculating the cooling of heating devices, taking into account changes in time of the temperature of the environment surrounding the heater $T_{e n v}=T_{e n v}(\tau)$, heat transfer coefficient $\alpha=\alpha(\tau)$, as well as the radiation temperature of the internal surfaces of fences $T_{\text {rad }}=T_{\text {rad }}(\tau)$.

One of the main areas of applicability of this method is the calculation of cooling of the building taking into account the heat storage capacity of heating devices in the event of an emergency shutdown of heat supply. The calculation methods currently used, described in $[14,15]$, do not take into account the heat storage of heating devices.

According to the computer simulation, when taking into account the heat storage capacity of heating devices (radiators), the cooling time of the room (building) changes depending on the outdoor temperature. A particularly significant effect is observed when the outdoor temperature is close to the average temperature of the heating period (which is mainly kept during the heating season), respectively, not taking into account the accumulated heat by the heating device would not be quite correct or at all inappropriate, if $t_{\text {outer }}>t_{\text {outer design }}$
Thecomplexityoftheproblemliesinthesimultaneouschangei ntimeofseveralparametersatonce.At the moment, there is no strict analytical solution to this problem. This paper provides an approximate analytical solution to the problem, as well as writing a methodology suitable for engineering calculations. The task is to find the surface temperature of the heater at any time and find the amount of heat that will give the heater over time $\tau$.

\section{MATERIALS AND METHODS}

Due to the fact that many heating devices are of the convective-radiation type, we will solve the problem with the condition that it transmits part of the heat by convection, and part by radiation.

To begin with, we will get a solution for an infinite plate. in such conditions, the mathematical formulation of the problem will take the form:

$$
\frac{\partial T}{\partial F_{O}}=\frac{\partial^{2} T}{\partial X^{2}}
$$

Where $X=\frac{x}{\delta}$ - dimensionless coordinate, at the accepted plate thickness $2 \delta, \quad F o=\frac{\alpha \tau}{\delta^{2}} \quad$ - Fourier number (dimensionless time), $a=\frac{\lambda}{c \rho}$ - thermal diffusivity, $\lambda$ conductivity coefficient, $c$ - specific heat, $\rho$ - density.

In general, we assume that the temperature change occurs only along one axis.

As an initial condition, we accept:

$$
T(X, 0)=f(X)
$$

Where $f(X)$ - even and continuous by $X$ function.

In general, the effect of radiation will be as follows:

$$
\left\{\begin{array}{l}
-\lambda \frac{\partial T}{\partial n}=\sigma\left(T^{4}(1, F o)-T_{\text {rad }}^{4}(F o)\right) \\
-\lambda \frac{\partial T}{\partial n}=\sigma\left(T^{4}(-1, F o)-T_{r a d}^{4}(F o)\right)
\end{array}\right.
$$

In this case, the temperature of the heating device will enter the boundary condition in the fourth degree, which will introduce non-linearity and, as a result, the absence of an analytical solution. In this case, we use linearization of boundary conditions. 
A.G. Gulkanov et al., International Journal of Emerging Trends in Engineering Research, 8(9), September 2020,6151 - 6156

Taking into account linearization, the boundary conditions will have the form:

$$
\begin{aligned}
&-\frac{\partial T(1, F o)}{\partial X}+B i(F o) {\left[T(1, F o)-T_{e n v}(F o)\right]+} \\
&+ 4 S k(F o)\left[T(1, F o)-T_{r a d}(F o)\right]=0 \\
& \frac{\partial T(-1, F o)}{\partial X}+B i(F o)\left[T(-1, F o)-T_{e n v}(F o)-\right]+ \\
&+4 S k(F o)\left[T(-1, F o)-T_{r a d}(F o)\right]=0
\end{aligned}
$$

Where $T_{e n v}$ - temperature of the environment around the heater, $B i\left(F_{o}\right)=\frac{\alpha(F o) \delta}{\lambda}-$ Bionumber, $\alpha\left(F_{O}\right)$ - heat transfer coefficient, $S k(F o)=\frac{\sigma T_{r a d}^{3}(F o) \delta}{\lambda}-$ Stark number, $\sigma$ Stefan-Boltzmann constant.

We will use the solution method according to [8], i.e. we will look for the solution of the problem in the form of the sum of the Poisson integral and two thermal potentials:

$$
\begin{aligned}
& T(X, F O)=\frac{1}{2 \sqrt{\pi}} \int_{-\infty}^{+\infty} \frac{1}{\sqrt{F O}} e^{-\frac{\left(X-X^{\prime}\right)^{2}}{4 F o}} g\left(X^{\prime}\right) d\left(X^{\prime}\right)+ \\
& +\frac{1}{\sqrt{\pi}} \int_{0}^{F o} \frac{\varphi_{1}\left(F O^{\prime}\right)}{\sqrt{F O-F O^{\prime}}} e^{-\frac{(X-1)^{2}}{4\left(F o-F o^{\prime}\right)}} d F O^{\prime}+ \\
& +\frac{1}{\sqrt{\pi}} \int_{0}^{F o} \frac{\varphi_{2}\left(F O^{\prime}\right)}{\sqrt{F O-F O^{\prime}}} e^{-\frac{(X+1)^{2}}{4\left(F o-F o^{\prime}\right)}} d F O^{\prime}
\end{aligned}
$$

Where $g(X)=f(X)$ in the range $-1 \leq X \leq 1$ and equal to $f(1)=f(-1)$ outside the specified interval, $\varphi_{1}(F o), \varphi_{2}(F o)$ the density of heatpotentials.Due to the property of the Poisson integral and thermal potentials, equation (6) is a solution of equation (1), taking into account the initial condition (2).

The property of thermal potentials is known from the equations of mathematical physics [1]:

$$
\lim _{X \rightarrow X_{0} \pm 0} \frac{\partial}{\partial X} \frac{1}{\sqrt{\pi}} \int_{0}^{F o} \frac{\varphi\left(F O^{\prime}\right)}{\sqrt{F O-F O^{\prime}}} e^{-\frac{\left(X-X_{0}\right)^{2}}{4\left(F o-F o^{\prime}\right)}} d F^{\prime}=\mp \varphi(F o)(7)
$$

Substituting (6) in the boundary conditions (4) and (5), taking into account the property of the thermal potential (7), we get that the condition will be fulfilled if the densities of the thermal potentials $\varphi_{1}(F o), \varphi_{2}(F o)$ satisfy a system of two Volterra equations:

$$
\begin{aligned}
& -\frac{\partial I(1, F o)}{\partial X}-\varphi_{1}(F o)+\frac{1}{\sqrt{\pi}} \int_{0}^{F o} \frac{\varphi_{2}\left(F o^{\prime}\right)}{\left(F o-F o^{\prime}\right)^{3 / 2}} e^{-\frac{1}{F o-F o^{\prime}}} d F o^{\prime}+ \\
& +B i(F o)\left[\begin{array}{l}
I(1, F o)+\frac{1}{\sqrt{\pi}} \int_{0}^{F o} \frac{\varphi_{1}\left(F o^{\prime}\right)}{\sqrt{F o-F o^{\prime}}} d F o^{\prime}+ \\
+\frac{1}{\sqrt{\pi}} \int_{0}^{F o} \frac{\varphi_{2}\left(F o^{\prime}\right)}{\sqrt{F o-F o^{\prime}}} e^{-\frac{1}{F o-F o^{\prime}}} d F o^{\prime}-T_{e n v}(F o)
\end{array}\right]+ \\
& 4 S k(F o)\left[\begin{array}{l}
I(1, F o)+\frac{1}{\sqrt{\pi}} \int_{0}^{F o} \frac{\varphi_{1}\left(F o^{\prime}\right)}{\sqrt{F o-F O^{\prime}}} d F O^{\prime}+ \\
+\frac{1}{\sqrt{\pi}} \int_{0}^{F o} \frac{\varphi_{2}\left(F o^{\prime}\right)}{\sqrt{F o-F o^{\prime}}} e^{-\frac{1}{F o-F o^{\prime}}} d F o^{\prime}-T_{r a d}(F o)
\end{array}\right]=0
\end{aligned}
$$

$$
\begin{aligned}
& -\frac{\partial I(-1, F o)}{\partial X}-\varphi_{2}(F o)+\frac{1}{\sqrt{\pi}} \int_{0}^{F o} \frac{\varphi_{1}\left(F O^{\prime}\right)}{\left(F o-F O^{\prime}\right)^{3 / 2}} e^{-\frac{1}{F o-F o^{\prime}}} d F O^{\prime}+ \\
& +B i(F o)\left[\begin{array}{l}
I(-1, F o)+\frac{1}{\sqrt{\pi}} \int_{0}^{F o} \frac{\varphi_{2}\left(F O^{\prime}\right)}{\sqrt{F o-F O^{\prime}}} d F O^{\prime}+ \\
+\frac{1}{\sqrt{\pi}} \int_{0}^{F o} \frac{\varphi_{1}\left(F O^{\prime}\right)}{\sqrt{F o-F O^{\prime}}} e^{-\frac{1}{F o-F o^{\prime}} d F O^{\prime}-T_{e n v}(F o)}
\end{array}\right]+ \\
& 4 S k(F o)\left[\begin{array}{c}
I(-1, F o)+\frac{1}{\sqrt{\pi}} \int_{0}^{F o} \frac{\varphi_{2}\left(F O^{\prime}\right)}{\sqrt{F o-F O^{\prime}}} d F O^{\prime}+ \\
+\frac{1}{\sqrt{\pi}} \int_{0}^{F o} \frac{\varphi_{1}\left(F O^{\prime}\right)}{\sqrt{F o-F O^{\prime}}} e^{-\frac{1}{F o-F o^{\prime}} d F O^{\prime}-T_{r a d}}
\end{array}\right]=0
\end{aligned}
$$

Where

$$
I(X, F O)=\frac{1}{2 \sqrt{\pi}} \int_{-\infty}^{+\infty} \frac{1}{\sqrt{F_{O}}} e^{-\frac{\left(X-X^{\prime}\right)^{2}}{4 F o}} g\left(X^{\prime}\right) d\left(X^{\prime}\right)
$$

- the Poisson integral.

Because $g(X)$ is an even function of $X \Rightarrow I\left(X, F_{O}\right)$ even $\Rightarrow \frac{\partial I(X, F o)}{\partial X}$ - odd function of $X$. Subtracting (9) from (8), we get that the difference of functions $\varphi_{1}-\varphi_{2}$ satisfies the Volterra integral equation $\Rightarrow \varphi_{1}-\varphi_{2} \equiv 0$

For simplicity we denote $\varphi_{1}=\varphi_{2}=\varphi$. Then we get that $\varphi$ satisfies the Volterra integral equation, i.e.:

$$
\varphi(F o)=\frac{1}{\sqrt{\pi}} \int_{0}^{F o} K\left(F o, F o^{\prime}\right) \varphi\left(F o^{\prime}\right) d F o^{\prime}+\psi(F o)
$$

Where

$$
\begin{aligned}
& \psi(F o)=-\frac{\partial I(1, F o)}{\partial X}+B i(F o)\left[I(1, F o)-T_{\text {env }}(F o)\right]+ \\
& +4 \operatorname{Sk}(F o)\left[I(1, F o)-T_{\text {rad }}(F o)\right]
\end{aligned}
$$

$K\left(F o, F o^{\prime}\right)$-the kernel of an integral operator defined by the expression:

$$
\begin{aligned}
& K\left(F O, F O^{\prime}\right)=\left(F O-F O^{\prime}\right)^{-3 / 2} e^{-\frac{1}{F o-F O^{\prime}}}+ \\
& +B i(F O)\left(F O-F O^{\prime}\right)^{-1 / 2}- \\
& +B i(F o)\left(F O-F O^{\prime}\right)^{-1 / 2} e^{-\frac{1}{F o-F o^{\prime}}} \\
& +4 S k(F O)\left(F o-F O^{\prime}\right)^{-1 / 2}- \\
& +4 S k(F o)\left(F o-F O^{\prime}\right)^{-1 / 2} e^{-\frac{1}{F o-F o^{\prime}}}
\end{aligned}
$$

By regrouping the members, we get:

$$
\begin{aligned}
& K\left(F O, F O^{\prime}\right)=B i(F O)\left(F o-F O^{\prime}\right)^{-1 / 2}+ \\
& +4 S k(F o)\left(F O-F O^{\prime}\right)^{-1 / 2}+ \\
& +\left(F o-F O^{\prime}\right)^{-1 / 2} e^{-\frac{1}{F o-F o^{\prime}}}\left[B i(F o)+4 S k(F O)+\frac{1}{F o-F O^{\prime}}\right]
\end{aligned}
$$


A.G. Gulkanov et al., International Journal of Emerging Trends in Engineering Research, 8(9), September 2020,6151 - 6156

\section{CALCULATING THE INTEGRAL EQUATION}

To simplify it, enter a new variable:

$\zeta=F o-F o^{\prime}$

Substituting (14) in (11), we rewrite the integral as the sum of several integrals and perform several algebraic transformations on each of them:

$$
\begin{gathered}
\int_{0}^{F o} B i(F o)\left(F o-F o^{\prime}\right)^{-1 / 2} \varphi\left(F o^{\prime}\right) d\left(F o^{\prime}\right)= \\
=B i(F o) \varphi(F o) \int_{0}^{F o} \zeta^{-1 / 2} d \zeta+ \\
+B i(F o) \int_{0}^{F o} \frac{\varphi(F o-\zeta)-\varphi(F o)}{\zeta} \zeta^{1 / 2} d \zeta \\
\int_{0}^{F o} 4 S k(F o)\left(F o-F o^{\prime}\right)^{-1 / 2} \varphi\left(F o^{\prime}\right) d\left(F o^{\prime}\right)= \\
=4 S k(F o) \varphi(F o) \int_{0}^{F o} \zeta^{-1 / 2} d \zeta+ \\
+4 S k(F o) \int_{0}^{F o} \frac{\varphi(F o-\zeta)-\varphi(F o)}{\zeta} \zeta^{1 / 2} d \zeta \\
\int_{0}^{F o} \varphi\left(F \sigma^{\prime}\right)\left(F o-F o^{\prime}\right)^{-1 / 2} e^{-\frac{1}{F o-F o^{\prime}}}\left[B i(F o)+4 S k(F o)+\frac{1}{F o-F o^{\prime}}\right. \\
=\int_{0}^{F o}\left[B i(F o)+4 S k(F o)+\frac{1}{\zeta}\right] \cdot \varphi(F o-\zeta) \cdot \zeta^{-1 / 2} e^{-1 / 5} d \zeta
\end{gathered}
$$

Combining equations (16), (17) and (18), we obtain a general equation for the integral in equation (11):

$$
\begin{aligned}
& \int_{0}^{F o} K\left(F o, F o^{\prime}\right) \varphi\left(F o^{\prime}\right) d F o^{\prime}=B i(F o) \varphi(F o) \int_{0}^{F o} \zeta^{-1 / 2} d \zeta+ \\
& +B i(F o) \int_{0}^{F o} \frac{\varphi(F o-\zeta)-\varphi(F o)}{\zeta} \zeta^{1 / 2} d \zeta+ \\
& +4 S k(F o) \varphi(F o) \int_{0}^{F o} \zeta^{-1 / 2} d \zeta+ \\
& +4 S k(F o) \int_{0}^{F o} \frac{\varphi(F o-\zeta)-\varphi(F o)}{\zeta} \zeta^{1 / 2} d \zeta+ \\
& +\int_{0}^{F o}\left[B i(F o)+4 S k(F o)+\frac{1}{\zeta}\right] \cdot \varphi(F o-\zeta) \cdot \zeta^{-1 / 2} e^{-1 / \zeta} d \zeta
\end{aligned}
$$

This representation was chosen in order to find an approximate analytical solution. It is obvious that the first and third integrals after the equal sign are elementary, and the rest can be found using approximate integration methods.

In the second and fourth integrals, the function is continuous, since:

$\lim _{\zeta \rightarrow 0} \frac{\varphi(F o-\zeta)-\varphi(F o)}{\zeta}=-\frac{\partial \varphi(F o)}{\partial F_{o}}(20)$

And also, by assumption, the function $\varphi(F o)$ is a differentiable function.
In the fifth integral:

$$
\lim _{\zeta \rightarrow 0}\left[B i(F o)+4 S k(F o)+\frac{1}{\zeta}\right] \varphi(F o-\zeta) e^{-1 / \zeta} \zeta^{-1 / 2}=0
$$

Thus, to find integrals in (19), we can apply approximate integration methods.

As part of the engineering problem, it is proposed to numerically find the values of functions that cannot be solved by analytical methods.

First let's look at the function $\psi(F o)$. Within the framework of the problem under consideration, it appears that the temperature field of the heating device is distributed evenly at the zero moment of time.

Then from (10) we will have:

$$
\begin{aligned}
& I(X, F o)=\frac{1}{2 \sqrt{\pi \cdot F O}} T_{\text {initial }} \int_{-\infty}^{+\infty} e^{-\frac{\left(1-X^{\prime}\right)^{2}}{4 F o}} d\left(X^{\prime}\right)= \\
& =\left.\frac{1}{2 \sqrt{\pi \cdot F O}} T_{\text {initial }} \cdot \lim _{\substack{a \rightarrow-\infty \\
b \rightarrow \infty}}\left(\sqrt{\pi \cdot F o} \cdot \operatorname{erf}\left(\frac{x-1}{2 \sqrt{F O}}\right)\right)\right|_{a} ^{b}= \\
& =T_{\text {initial }}
\end{aligned}
$$

Where $T_{\text {initial }}$ - the initial temperature of the heater.

Hence:

$$
\frac{\partial I(1, F o)}{\partial X}=0
$$

Then taking into account (22), (12) will take the form:

$$
\begin{aligned}
\psi(F o)=B i(F o)\left[T_{\text {initial }}-T_{\text {env }}(F o)\right]+ \\
+4 S k(F o)\left[T_{\text {initial }}-T_{\text {rad }}(F o)\right]
\end{aligned}
$$

Now let's go to the $\varphi(F o)$ value. To solve this problem, we will use classical methods of approximate integration. We will look for a solution (11) in the interval $F o \in(0, N)$. Divide the interval $(0, N)$ into $n$ equal parts. $F o_{i}=\frac{N}{n} i, i=0,1,2, \ldots, n$ .Then we get a system of $(n+1)$ equations with $(n+1)$ unknowns $\varphi\left(F_{O_{i}}\right)$.

$$
\left\{\begin{array}{l}
\varphi(0)=\psi(0) \\
\varphi\left(F o_{i}\right)\left(\sqrt{\pi}+2 B i\left(F o_{i}\right) F o_{i}^{1 / 2}\right)+ \\
+i \sum_{k=1}^{i} M_{i}^{k} F o_{k}^{1 / 2} \cdot B i\left(F o_{i}\right) \frac{\varphi\left(F o_{i}\right)-\varphi\left(F o_{i-k}\right)}{k}+ \\
+\varphi\left(F o_{i}\right)\left(\sqrt{\pi}+8 S k\left(F o_{i}\right) F o_{i}^{1 / 2}\right)+ \\
+i \sum_{k=1}^{i} M_{i}^{k} F o_{k}^{1 / 2} \cdot 4 S k\left(F o_{i}\right) \frac{\varphi\left(F o_{i}\right)-\varphi\left(F o_{i-k}\right)}{k}+(25) \\
+i \sum_{k=1}^{i} M_{i}^{k} F o_{k}^{1 / 2} \varphi\left(F o_{i-k}\right) e^{-\frac{1}{F o_{k}}} \cdot \\
B i\left(F o_{i}\right)+4 S k\left(F o_{i}\right)+\frac{1}{F o_{k}}=\sqrt{\pi} \psi\left(F o_{i}\right) \\
\cdot \frac{k}{i=1,2,3, \ldots, n}
\end{array}\right.
$$


A.G. Gulkanov et al., International Journal of Emerging Trends in Engineering Research, 8(9), September 2020,6151 - 6156

Here $M_{i}^{k}$ - are coefficients determined depending on the integration method (trapezoid method, Simpson method, etc.)

\section{DETERMINING THE TEMPERATURE OF THE HEATING DEVICE}

Rewrite (6) in a different form, with the condition of replacement $F o-F O^{\prime}=\zeta$, and also $\varphi_{1}=\varphi_{2}=\varphi$ :

$$
\begin{aligned}
& T(X, F O)=I(X, F O)+\frac{1}{\sqrt{\pi}} \int_{0}^{F o} \frac{\varphi(F o-\zeta)}{\zeta^{1 / 2}} e^{-\frac{(1-X)^{2}}{4 \zeta}} d \zeta+ \\
& +\frac{1}{\sqrt{\pi}} \int_{0}^{F o} \frac{\varphi(F o-\zeta)}{\zeta^{1 / 2}} e^{-\frac{(1+X)^{2}}{4 \zeta}} d \zeta
\end{aligned}
$$

Since $I(X, F o)$ - even function of $X$, then $T(X, F o)$ it will also be an even functionof $X$, it follows that it is sufficient to consider (26) when $X \in(0,1)$. The function $\varphi(F o)$ was defined by us in the third point with its discrete values $\varphi\left(F o_{i}\right)$.However, the application of approximate integration methods directly to integrals in (26) is unacceptable, since $X=1$ the first integral is improper. Then consider $X<1$, then for simplicity we denote $\varepsilon=1-X$.

We transform the first integral in (26) to the form:

$$
\begin{aligned}
& \frac{1}{\sqrt{\pi}} \int_{0}^{F o} \frac{\varphi(F o-\zeta)}{\zeta^{1 / 2}} e^{-\frac{(1-X)^{2}}{4 \zeta}} d \zeta= \\
& \frac{1}{\sqrt{\pi}} \int_{0}^{F o} \frac{\varphi(F o-\zeta)-\varphi(F o)}{\zeta} \zeta^{1 / 2} e^{-\frac{(1-X)^{2}}{4 \zeta}} d \zeta+
\end{aligned}
$$

$+\varphi(F o) \cdot \frac{1}{\sqrt{\pi}} \int_{0}^{F o} \zeta^{-1 / 2} e^{-\frac{(1-X)^{2}}{4 \zeta}} d \zeta$

Consider the first integral in (27). The continuity of the first multiplier was proved earlier. It is obvious that the function $\zeta^{1 / 2} e^{-\frac{(1-X)^{2}}{4 \zeta}}$ is continuous throughout the entire half-plane $\zeta \geq 0$, hence it follows that approximate integration methods are applicable to the first integral.

The second integral in (27) is calculated analytically:

$$
\begin{aligned}
& I_{1}=\frac{1}{\sqrt{\pi}} \int_{0}^{F o} \zeta^{-1 / 2} e^{-\frac{\varepsilon^{2}}{4 \zeta}} d \zeta=\frac{2 \sqrt{F O}}{\sqrt{\pi}} e^{-\frac{\varepsilon^{2}}{4 F o}}+ \\
& +\varepsilon\left(\operatorname{erf}\left(\frac{\varepsilon}{2 \sqrt{F O}}\right)-1\right)=\frac{2 \sqrt{F O}}{\sqrt{\pi}} e^{-\frac{(1-X)^{2}}{4 F o}}+ \\
& +(1-X)\left(\operatorname{erf}\left(\frac{1-X}{2 \sqrt{F O}}\right)-1\right)
\end{aligned}
$$

The error function is unrepresentable via elementary functions, but it is possible to decompose the integrable expression into a Taylor series, and by integrating it termwise, we can get its representation as a series:

$\operatorname{erf}\left(\frac{1-X}{2 \sqrt{F_{O}}}\right)=\frac{2}{\sqrt{\pi}} \sum_{n=0}^{\infty} \frac{(-1)^{n}\left(\frac{1-X}{2 \sqrt{F_{O}}}\right)^{2 n+1}}{n !(2 n+1)}$

Thus, the temperature can be calculated using the formula: $T(X, F o)=I(X, F o)+\varphi(F o) I_{1}(X, F o)+$ $+I_{2}(X, F o)+I_{3}(X, F o)$

Where

$$
\begin{aligned}
& I_{2}=\frac{1}{\sqrt{\pi}} \int_{0}^{F o} \frac{\varphi(F o-\zeta)-\varphi(F o)}{\zeta} \zeta^{1 / 2} e^{-\frac{(1-X)^{2}}{4 \zeta}} d \zeta \\
& I_{3}=\frac{1}{\sqrt{\pi}} \int_{0}^{F o} \frac{\varphi(F o-\zeta)}{\zeta^{1 / 2}} e^{-\frac{(1+X)^{2}}{4 \zeta}} d \zeta
\end{aligned}
$$

The values (31) and (32) can be found using approximate integration methods:

$$
\begin{aligned}
& I_{2}(X, F O)=\frac{i}{\sqrt{\pi}} \sum_{k=1}^{i} M_{i}^{k} \frac{\varphi\left(F O_{i-k}\right)-\varphi\left(F O_{i}\right)}{k} \cdot F O_{k}^{1 / 2} e^{-\frac{(1-X)^{2}}{4 F o_{k}}} \\
& I_{3}(X, F O)=\frac{i}{\sqrt{\pi}} \sum_{k=1}^{i} M_{i}^{k} \frac{\varphi\left(F O_{i-k}\right)}{k} \cdot F O_{k}^{1 / 2} e^{-\frac{(1+X)^{2}}{4 F o_{k}}}
\end{aligned}
$$

But, as it was written earlier, the resulting solution (30) describes only a one-dimensional non-stationary temperature field in an infinite plate. To find the non-stationary temperature field of objects of finite dimensions, we need to use the multiplication theorem of solutions. The theorem states that the dimensionless temperature field of a complex object is equal to the product of the dimensionless temperature fields of the corresponding primitives. In the framework of this problem, the intersection of three plates of different thickness will give the desired solution, i.e.:

$$
\begin{aligned}
& \frac{T(x, y, z, \tau)-T_{0 . e n v}}{T_{0}-T_{0 . e n v}}=\frac{T(x, \tau)-T_{0 . e n v}}{T_{0}-T_{0 . e n v}} \cdot \frac{T(y, \tau)-T_{0 . e n v}}{T_{0}-T_{0 . e n v}} . \\
& \cdot \frac{T(z, \tau)-T_{0 . e n v}}{T_{0}-T_{0 . e n v}}
\end{aligned}
$$

It is known that this solution is only applicable at a constant ambient temperature, but since the temperature of the air surrounding the heater varies in a small range, this solution can be applied.

In this case, the thickness of the plate is each of the geometric dimensions of the heater.

De-dimensioning equation (30), taking into account (22), and similarly writing the values $\theta_{Y}$ and $\theta_{Z}$, we get:

$$
\begin{aligned}
& \theta_{X}=\frac{T(X, F O)-T_{0 . e n v}}{T_{\text {initial }}-T_{0 . e n v}}=\frac{T_{\text {initial }}+\varphi(F O) I_{1}(X, F O)}{T_{\text {initial }}-T_{0 . e n v}}+ \\
& +\frac{I_{2}(X, F O)+I_{3}(X, F O)-T_{0 . e n v}}{T_{\text {initial }}-T_{0 . e n v}} \\
& \theta_{Y}=\frac{T(Y, F O)-T_{0 . e n v}}{T_{\text {initial }}-T_{0 . e n v}}=\frac{T_{\text {initial }}+\varphi(F O) I_{1}(Y, F O)}{T_{\text {initial }}-T_{0 . e n v}}+ \\
& +\frac{I_{2}(Y, F O)+I_{3}(Y, F O)-T_{0 . e n v}}{T_{\text {initial }}-T_{0 . e n v}}
\end{aligned}
$$


A.G. Gulkanov et al., International Journal of Emerging Trends in Engineering Research, 8(9), September 2020,6151 - 6156

$$
\begin{aligned}
& \theta_{Z}=\frac{T(Z, F o)-T_{0 . e n v}}{T_{\text {initial }}-T_{0 . e n v}}=\frac{T_{\text {initial }}+\varphi(F o) I_{1}(Z, F o)}{T_{\text {initial }}-T_{0 . e n v}}+ \\
& +\frac{I_{2}(Z, F o)+I_{3}(Z, F o)-T_{0 . e n v}}{T_{\text {initial }}-T_{0 . e n v}}
\end{aligned}
$$

-the dimensionless coordinate, where $2 \delta_{1}$ - height of the heater, $Z=\frac{z}{\delta_{2}}$ - the dimensionless coordinate, where $2 \delta_{2}$ width of the heater.

Then the dimensionless temperature of the heater can be found as:

$$
\theta=\theta_{X} \theta_{Y} \theta_{Z}
$$

Now calculate the temperature of the wall of the heater.

Differentiating by $X$ (26), we will have:

$\frac{\partial T(1, F o)}{\partial X}=\frac{\partial I(1, F o)}{\partial X}+\varphi(F o)-$

$-\frac{1}{\sqrt{\pi}} \int_{0}^{F o} \frac{\varphi(F o-\zeta)}{\zeta^{3 / 2}} e^{-\frac{1}{\zeta}} d \zeta$

Then, taking into account the boundary condition (4), the solution takes the form:

$$
\begin{aligned}
& \frac{\partial I(1, F o)}{\partial X}+\varphi(F o)-\frac{1}{\sqrt{\pi}} \int_{0}^{F o} \frac{\varphi(F o-\zeta)}{\zeta^{3 / 2}} e^{-\frac{1}{\zeta}} d \zeta= \\
& =B i(F o)\left[T(1, F o)-T_{c p}(F o)\right]+ \\
& +4 S k(F o)\left[T(1, F o)-T_{r a d}(F o)\right]
\end{aligned}
$$

By expressing $T(1, F o)$ explicitly, given (23), we get an expression for the temperature of an infinite plate, i.e.:

$$
\begin{aligned}
& T(1, F o)=\frac{B i(F o) T_{e n v}(F o)+4 S k(F o) T_{r a d}(F o)}{B i(F o)+4 S k(F o)}+ \\
& +\frac{\varphi(F o)-\frac{1}{\sqrt{\pi}} \int_{0}^{F o} \frac{\varphi(F o-\zeta)}{\zeta^{3 / 2}} e^{-\frac{1}{\zeta}} d \zeta}{B i(F o)+4 S k(F o)}
\end{aligned}
$$

De-dimensionalization of the equation (42), we get:

$$
\begin{aligned}
& \theta_{X}=\frac{B i(F o) T_{e n v}(F o)-T_{0 . e n v}}{(B i(F o)+4 S k(F o))\left(T_{0}-T_{0 . e n v}\right)}+ \\
& +\frac{4 S k(F o) T_{\text {rad }}(F o) \cdot \varphi(F o)-\frac{1}{\sqrt{\pi}} \int_{0}^{F o} \frac{\varphi(F o-\zeta)}{\zeta^{3 / 2}} e^{-\frac{1}{\zeta}} d \zeta}{(B i(F o)+4 S k(F o))\left(T_{\text {initial }}-T_{0 . e n v}\right)} \\
& \text { Where } \theta_{X}=\frac{T(1, F o)-T_{\text {initial }}}{T_{\text {initial }}-T_{0 . \text { env }}} .
\end{aligned}
$$

Also, to find the temperature of the wall of the heater, you need to know the temperature functions for two other coordinates, and in general these coordinates are not equal to one. $\theta_{Y}$ and $\theta_{Z}$ were defined by us above.Then the dimensionless temperature of the heater wall can be found by the formula (39).

\section{THE AMOUNT OF HEAT RELEASED FROM THE HEATING DEVICE}

The amount of heat released from the heater during $\tau$ can be found:

$$
Q=c m\left(T_{\text {initial }}-T_{0 . e n v}\right)(1-\bar{\theta})
$$

Where $c$ - specific heat capacity of the material; $m$ - the weight of the heater;

$\bar{\theta}$ - the average dimensionless temperature of the heating device over time $\tau$. The average value can be found using the formula:

$$
\bar{\theta}=\int_{0}^{1} \theta_{X} d X \cdot \int_{0}^{1} \theta_{Y} d Y \cdot \int_{0}^{1} \theta_{Z} d Z(45)
$$

\section{BRINGING THE PROBLEM TO A MORE PRECISE GEOMETRY}

The geometry described above describes a heating device as a parallelepiped. in some problems, this representation is not applicable, because such a description will not show the exact values of the desired parameters, so it is proposed to consider a real heating device as a set of corresponding primitives, for which an analytical solution is obtained. For example, consider a model of a cast-iron radiator.

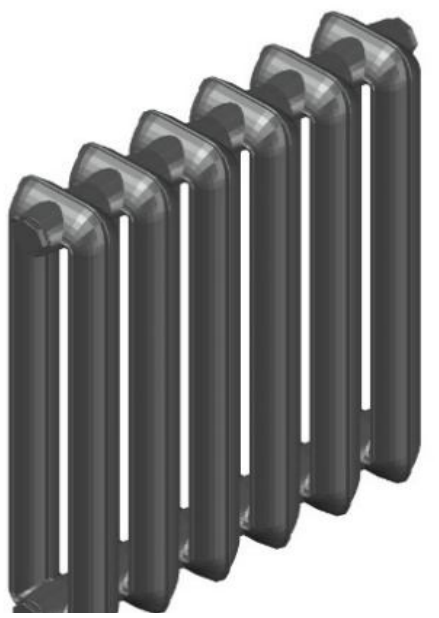

Figure 1: Model of a real heating device.

This geometry can be reduced to a simpler one consisting of parallelepipeds. This geometry will look like:

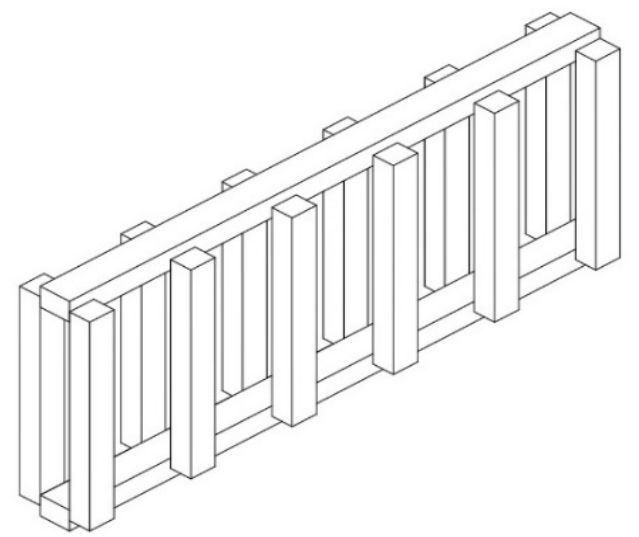

Figure 2: Model of the heating device accepted for calculations. 
This geometry will describe the task much more accurately. Thus, a similar technique can be applied to other types of heating devices, including bimetallic radiators.

\section{CONCLUSION AND DISCUSSIONS}

In this paper, the process of cooling a heating device at a variable internal temperature, heat transfer coefficient, and variable radiation temperature of the internal surfaces of fences was considered using mathematical methods specified in [1-7]. In addition to the solution found by analytical methods, a method suitable for engineering calculations was obtained.

As a continuation of the study, it is proposed to take into account, in addition to all the factors taken into account, the mobility of indoor air due to convective flows.

\section{REFERENCES}

1. A. N. Tikhonov, A. A. Samarsky, Equations of mathematical physics. Published by the home edition of physical and mathematical literature of «Science» publishing house, 1977.

2. N. S. Koshlyakov, E. B. Gliner, M. M. Smirnov, Partial differential equations of mathematical physics. Published by publishing house «Higher school», 1970.

3. B. P. Demidovich, I. A. Maron, Fundamentals of computational mathematics. Published by main editorial office of physical and mathematical literatureof «Science» publishing house, 1966.

4. R. P. Fedorenko, Introduction to computational physics. Published by "Intelligence" publishing house, 2008.

5. A. A. Samarskii, A. V. Gulin, Numerical methods. Published by main editorial office of physical and mathematical literature of «Science» publishing house, 1989.

6. E. M. Kartashov, Analytical methods in the theory of thermal conductivity of solids. Published by publishing house «Higher school», 2001.

7. A.V. Lykov, «Theory of thermal conductivity», Published by publishing house «Higher school», 1967.

8. K. A. Kiselev, A. I. Lazarev, «Temperature field of an unlimited plate at a variable value of the heat transfer coefficient and a variable ambient temperature», Journal of technical physics, Vol. 30, №6, PP. 616-621, 1960

9. Yu. L. Rosenstock, «Temperature field of an unlimited plate under conditions of dependence of the ambient temperature and heat transfer coefficient on time», Engineering and physical journal, Vol. 6, №3, PP. 45-50, 1963
10. A. L. Burka, «Asymmetric radiant-convective heating of an unlimited plate», Applied mechanics and technical physics, №2, 1966

11. Yu. V. Vidin, V. V. Ivanov «Calculation of asymmetric heating of an unlimited plate under the influence of radiation», News of Higher Educational Institutions. Ferrous metallurgy. №12, PP 144-147, 1964

12. Yu. V. Vidin«On the temperature field of an unbounded plate with a variable heat exchange coefficient», News of Higher Educational Institutions. Aviation equipment, №1, PP 65-69, 1967

13. Yu. V. Vidin, V. V. Ivanov «On the temperature field in solids when heated simultaneously by radiation and convection»,News of Higher Educational Institutions. Ferrous metallurgy. №5, PP 143-146, 1967

14. V. N. Bogoslovsky, A. N. Skanavi, Heating. Published by «Stroizdat» publishing house, 1991.

15. E. Ya. Sokolov, Heating and heat networks. Published by MEI publishing house, 2001.

16. N.Mukilarasan, R.Karthikeyan «Experimental Thermal evaluation of Fluidized Bed Cooling Tower (FBCT) with various Bed elevations and Spherical turbulent balls», International Journal of Emerging Trends in Engineering Research, Vol. 8, №8, PP. 4091-4096, 2020

17. S.M. Usikov«Simulation of Heat Transfer by Water Heating Convectors», International Journal of Emerging Trends in Engineering Research, Vol. 8, №3, PP. 726-734, 2020

18. V.D. Makarishev, G.A. Savenko, S.M. Usikov« Determination of the Optimal Design of a Thermal Panel of an Autonomous Residential Module by Calculating Temperature Field», International Journal of Emerging Trends in Engineering Research, Vol. 8, №4, PP. 1217-1221, 2020 